\title{
A Probabilistic Model for Characterising Heat Pump Electrical Demand versus Temperature
}

\author{
Amy Anderson, Bruce Stephen, Rory Telford, Stephen McArthur \\ Electronic \& Electrical Engineering \\ University of Strathclyde \\ Glasgow, UK \\ amy.anderson@strath.ac.uk, bruce.stephen@strath.ac.uk, rory.telford@strath.ac.uk, s.mcarthur@strath.ac.uk
}

\begin{abstract}
The introduction of electric heat pumps as an alternative to gas based systems for space heating offers a potential pathway for reducing the carbon emissions produced to meet domestic heating demand in the UK. The adoption of heat pumps has the potential to significantly re-shape typical domestic load profiles, however uptake within the UK is currently limited and the effects of wide-scale adoption on distribution networks is not well understood. Heat pump demand is highly sensitive to temperature, lessening load profile diversity, but is also influenced by behavioral routine and is therefore not entirely deterministic. This study develops a probabilistic demand model from real customer heat pump data for translating electrical heat pump demand/air temperature relations to account for regional variation. A $L V$ network heat pump penetration study is performed to demonstrate how residential network impact can be assessed using the model.
\end{abstract}

Keywords-heat pumps, low carbon technologies, load modelling, uncertainty

\section{INTRODUCTION}

The reduction of the contribution of heat to the UK's overall greenhouse gas emissions currently presents one of the largest challenges to achieving the long-term emissions targets set by UK government policy. Half of final UK energy consumption consumed in the UK is to provide heating, greater than the energy consumed for electricity or transport [1]. The contribution of domestic heating is estimated to form $32 \%$ of average UK household greenhouse gas emissions [2]; in order to achieve 2050 Net-zero goals this must be reduced by a further $95 \%$ from 2017 levels [2]. Achieving the target decarbonisation of the UK's heating sector therefore requires a radical shift in the technological status quo, necessitating widespread adoption of low carbon heating solutions in combination with improved efficiency measures.

Electric Heat Pumps (EHP) offer one potential low carbon alternative to conventional heating options; reductions in $\mathrm{CO} 2$ emissions of up to $25 \%$ per unit of heat generated are achievable [3]. In combination with a fully renewable electricity source, this can reduce the effective $\mathrm{CO} 2$ emissions required for a household due to heating to zero. Whilst not suitable for all households, this technology has been identified as a low-regret option for households not presently connected to the main gas network within the UK [4], in addition to being a possible lowest cost option for new or existing wellinsulated households.

The incorporation of heat pumps onto LV networks poses many challenges for distribution network operators (DNOs) The instantaneous electrical demand of a typically sized domestic heat pump can be equivalent or in excess of current daily domestic demand peaks [5]. Heat pump routines are anticipated to follow similar behaviour to current conventional heating demand patterns, with heating typically switched off when occupants are asleep or not at home [6]. This low temporal diversity in combination with high peak demand means that a high network penetration of heat pumps has the potential to put a significant strain on existing LV network assets and incur costly network reinforcement to maintain existing quality of service for customers.

The growth of heat pump technology has been supported by UK government policy [7] and industry trials [8] but despite this, overall deployment remains low - 76,388 domestic heat pumps were registered with the RHI (Renewable Heat Incentive) scheme in the UK by the end of 2019 [9]. This results in a general lack of operational demand data that can be used to facilitate heat pump effects analysis. Analysis and prediction of heat pump electrical demand modelling within the UK is therefore currently constrained in the literature primarily to either small-scale physical models which require a high-level of specific system knowledge to make predictions [10], or methods which rescale existing heat pump trial data to achieve a deterministic outcome [11].

Navarro-Espinosa et al. developed a high-resolution probabilistic model for demand prediction which draws on operational data from 72 micro-CHP (combined heat and power) units during field trials in 2011 [12]. However, this study is reliant on the fact that micro-CHP technology represents a good approximation of EHP demand patterns, and does not draw on real EHP operational data. The probabilistic methodology proposed by this paper, however, features many benefits for LV network operators in that the probability of a different range of values are defined, rather than predicting a fixed demand plus error for certain operating conditions.

Recent UK trials have greatly improved the availability of domestic demand EHP data [13] [14]. This work seeks to build on the probabilistic approach of previous heat pump studies and leverage the most recent data to characterise the electrical demand of a heat pump versus temperature. Physically this relationship is determined by the heat pump rating, in combination with efficiency and specific building parameters. Electrical demand is also influenced by behavioural effects that are difficult to capture suitably in a physics based model. This paper will describe a translational method that offers middle ground between required knowledge of the system whilst retaining the uncertainty inherent in real data. Furthermore, metrics are developed in order to quantify the uncertainty around a prediction in order to translate demand forecasts into a usable output.

\section{METHOD}

This paper describes a method for probabilistically generating synthetic daily electrical demand for a single heat pump in isolation or across a population, with the characteristics derived from real data. The magnitude of heat pump demand is sensitive to outdoor temperature, but is also determined by relatively fixed parameters such as building type and insulation, as well as variable user behaviours. This model will utilise UK domestic heat pump data coupled with historical weather data in order to characterise the 
fundamental relationship between daily electrical demand and temperature for heat pumps. The two primary elements of this study used to generate predictions are:

- Demand characterisation; extraction of the electrical heat pump demand temperature relationship from operational data

- Population characterisation; characterisation of the distribution of heat pump rating across the sample dataset such that new artificial populations can be generated

These relationships are then combined with weather data and a user-defined number of households in order to make predictions for cases independent of the original sample dataset. The interrelationships between model data, characterisation and model tests for this work are defined in Fig 1. The datasets used for developing this model are described in more detail in Section IIA.

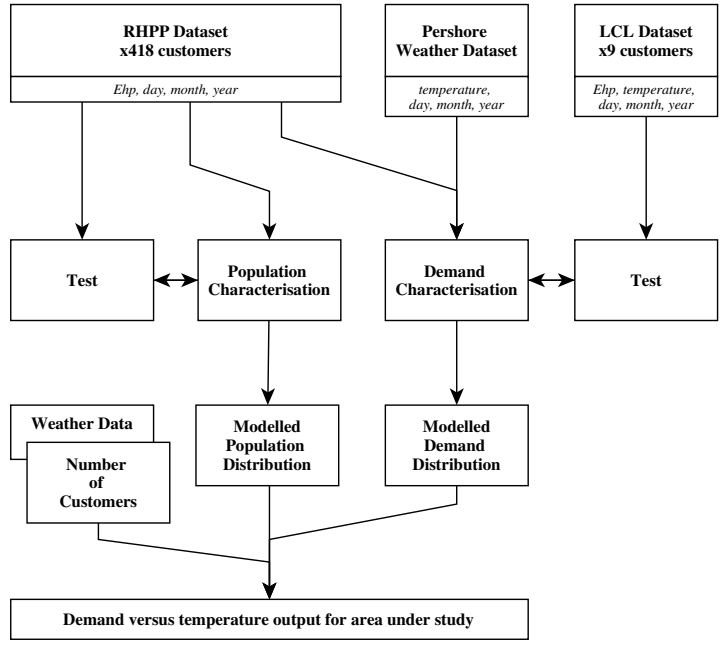

Fig. 1. Model Overview

\section{A. Datasets}

This work makes use of three datasets to model the relationships between demand, temperature, and population size. There is presently no large-scale heat pump dataset available that sufficiently captures all of these parameters; therefore different data sources are combined in order to define the relationship between heat pump electrical demand and temperature.

The Renewable Heat Premium Payment (RHPP) dataset [15] features high-resolution 2-minutely electrical demand data collected for 418 air source and ground source heat pumps from October 2013 to March 2015. This dataset does not feature location data or local weather measurements; this necessitates the development of a method for leveraging the high-resolution RHPP demand measurements whilst retaining the demand relationship with respect to temperature.

Historical weather data from the Centre for Environmental Data Analysis (CEDA) is mapped to the RHPP dataset [16]. The weather station at Pershore has been selected; this was initially selected due to its use as one of the benchmark weather stations for generation of the Central England temperature dataset. Furthermore, this weather is close to the population centre of the UK and is therefore on average representative of UK weather for the majority of inhabitants.

Finally, the operational data collected during the Low Carbon London (LCL) heat pump trials [13] is used for validation purposes. This dataset features electrical heat pump demand versus local temperature measurements for nine customers; this data is used to validate that mapping the RHPP dataset versus an artificial weather station results in an acceptable accuracy. Customers 1 to 5 and 7 feature two years worth of data; the remaining customers 6,8 and 9 only feature 60 days of data. All customers have air-source heat pumps installed with heat pump sizes ranging from $8-16 \mathrm{~kW}$.

\section{B. Demand Characterisation}

Electric heat pump daily demand is broadly proportional to temperature; lower ambient temperatures translates into higher heat pump demand, and vice versa for high ambient temperatures. The influence of parameters such as heat pump rating, efficiency, building insulation type and most importantly behavioural parameters result in a range of possible values given a single daily average temperature measurement rather than a single possible value. This is directly observable in the Low Carbon London dataset shown in Fig. 2, with a particularly wide band of possible demand values for $10{ }^{\circ} \mathrm{C}$. Customers 6,8 and 9 are reduced datasets only featuring 60 days of data and therefore only show a partial illustration of this characteristic - they do not capture the full operational variation due to seasonal changes.

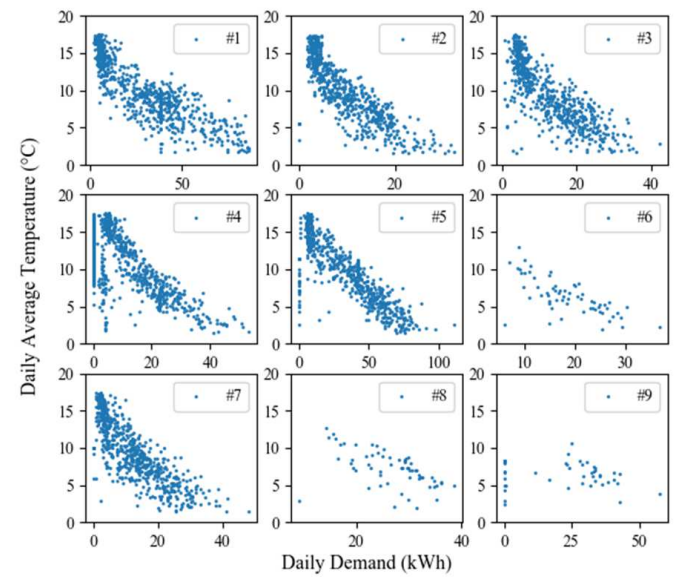

Fig. 2. Daily demand versus daily average tempeature for Low Carbon London heat pump customers \#1 to \#9

In order to create the basis for the probabilistic model, the demand for each RHPP customer is converted from an hourly to daily demand figure rated in $\mathrm{kWh}$. In order to allow for comparison across the entire dataset, the daily demand figure for each customer is normalised using the formula:

$$
D_{\text {normalised }}=\frac{D_{k W h}}{D \max }
$$

where $D_{k W h}$ is the daily demand for a specific day and $D \max$ is the maximum daily demand for the customer dataset being normalised. This scales all customer data on a range from 0 to 1; 0 representing zero demand and 1 representing maximum demand. The normalised customer demand is mapped to the CEDA MIDAS Pershore weather station data for the same time period as the RHPP set. The RHPP customer demand data and weather data is then unified and plotted in the heatmap shown in Fig. 3. The aggregated demand data has 
been split into $1{ }^{\circ} \mathrm{C}$ intervals and the distribution plotted in Fig. 3. This clearly illustrates the same characteristic shape as the Low Carbon London data; a narrow tail for ambient temperatures above $15{ }^{\circ} \mathrm{C}$ and a widening band of higher demand for lower temperatures. Datapoints for lower temperatures are sparse in the overall dataset and the increased granularity of the distribution at very low temperatures is visible.

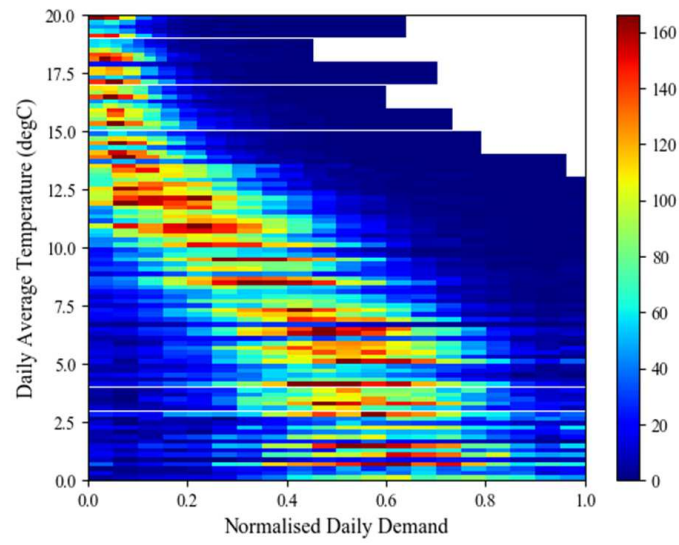

Fig. 3. Implied dependency structure of normalised heat pump daily demand versus daily average temperature $\left({ }^{\circ} \mathrm{C}\right)$ from RHPP dataset

The standard deviation and mean is calculated for each of the $1{ }^{\circ} \mathrm{C}$ dataset intervals, translating the raw data into a simplified Gaussian distribution for each temperature band. This provides a probabilistic range of normalised demand values possible for each $1{ }^{\circ} \mathrm{C}$ interval. This is represented in (2) as:

$$
f_{t}(x)=\frac{1}{\sigma_{t} \sqrt{2 \pi}} e^{-\frac{1}{2}\left(\frac{x-\mu_{t}}{\sigma_{t}}\right)^{2}}
$$

Therefore when making a demand prediction the daily average temperature is first identified, and then the demand value is generated based on a distribution determined by the corresponding standard deviation and mean for that temperature that has been derived from the data in Fig.3. This allows for the creation of a look up table of standard deviation and mean values for each temperature. In order to translate the normalised demand back into $\mathrm{kWh}$ the formula shown in (3) is used.

$$
D_{\text {Predicted }(k W h)}=f_{t}(x) \times \frac{\bar{D}_{k W h}}{\bar{D}_{\text {Normalised }}}
$$

\section{Population Characterisation}

The RHPP dataset is analysed to quantify the distribution of heat pump rating amongst the overall population. This is not directly inferable from the available metadata. Furthermore the final electrical demand recorded is a function of heat pump, building characteristics and behavioural elements. Therefore the aim is to characterise the overall population distribution such that the distribution may be resized for smaller and larger population sets. In order to apply (3) the mean daily $\mathrm{kWh}$ value for each customer in the dataset is calculated, allowing for the creation of new artificial populations. Users are randomly selected from the population shown in Fig.4 when creating demand profiles. The limitations of this method have been identified in other papers - customers in the RHPP dataset are predominately-council houses rather than private tenants [11]. Additionally, heat pump technology has advanced since the installation of the sample population hardware in 2013 and therefore this may not fully be representative of a modern population.

The median maximum daily demand for this population is $32.2 \mathrm{kWh}$. This aligns with [11], which predicts a daily demand of approximately $31.2 \mathrm{kWh}$ for a cold winter weekday from the same RHPP dataset. In contrast, the averaged daily maximum from the Customer Led Network Revolution (CLNR) dataset consumed $13.96 \mathrm{kWh}$ [17]. As this is an average daily figure it does not directly compare to the RHPP peak daily figure; however the greater than $50 \%$ difference between the two daily demand values does indicate that the sampled population dataset will have a strong influence on the final predicted demand outcomes.

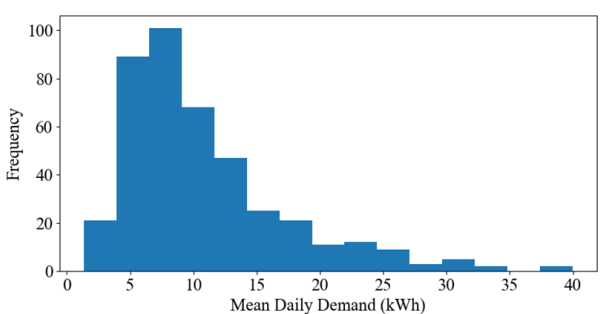

Fig. 4. Distribution of RHPP Customers by mean heat pump daily demand $(\mathrm{kWh})$

\section{Model Validation}

The simplification of the total customer dataset into a single common demand model requires several points to be validated prior to further application. A single UK temperature dataset has been used for the 418 distinct RHPP customers; the models ability to predict demand versus temperature must be validated. Furthermore the normalisation process performed to fit all RHPP customers to the same scale must be proved to be reversible in order to allow for final prediction of demand in $\mathrm{kWh}$.

Both of these points are tested by applying the model derived from RHPP data to the LCL customer data. The LCL customer data features local temperature measurements and real demand data, allowing for the modelled temperature versus demand relationship to be tested. The normalisation process is tested by detecting the median daily demand for each customer and using this to translate the normalised prediction into $\mathrm{kWh}$ as defined in (3).

\section{Model Validation Results}

The predicted demand derived from the RHPP dataset is plotted versus the real demand of Low Carbon London customers in Fig.5. The only two inputs required in order to predict the demand is the weather data featured in the LCL dataset in combination with the median sampled real demand and predicted demand in (3) to act as a scaling factor between the normalised and real demand figures; beyond this the LCL dataset is independent of the RHPP model. A moving average of 10 days has been applied in order to reduce noise when plotting the graphs, but this moving average has not been used in the later MAE error calculations. There are several instances in the plotted real data where the demand remains fixed at zero or another intermediate value for several successive days; these instances have been retained for the plots in order to highlight the capability of the predictive model. 
There are two levels of abstraction between the real LCL data and the derived RHPP model. Firstly the LCL data is assumed to be normally distributed for each $1 \mathrm{degC}$ temperature slice. Beyond this, the simplified normal distribution for each set of customer data features another error between the Gaussian distribution generated from the real data and the Gaussian distribution set derived from the RHPP customer data.

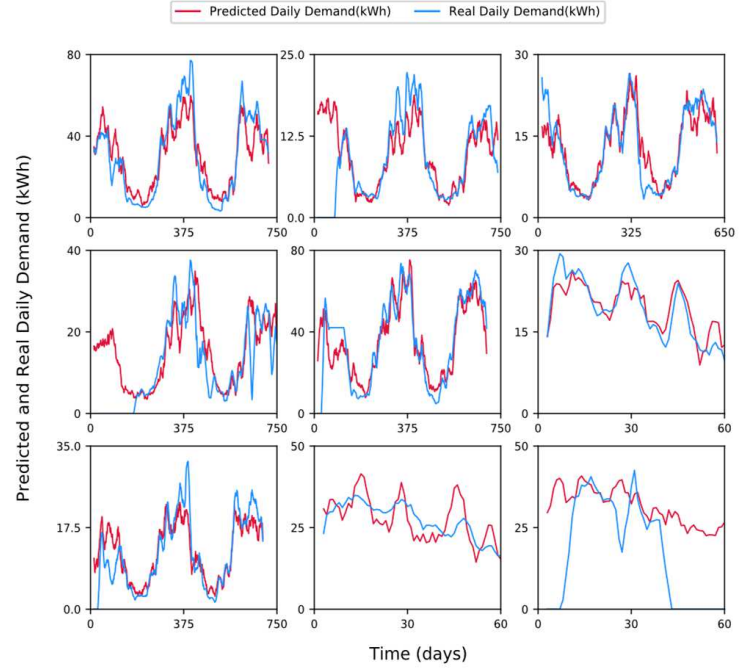

Fig. 5. Predicted daily demand derived from Gaussian distribution lookup table versus real Low Carbon London demand $(\mathrm{kWh})$ for nine customers

Table 1 presents an overview of the predictive model's scores for the nine LCL customers. The mean demand is compared with the MAE as an absolute and percentage figure, with the maximum real demand provided for further context of the magnitude of the MAE. When calculating this figure any real data where the demand is zero for more than five consecutive measurements has been discarded as these are assumed to be periods when the monitoring equipment rather than the heat pump was inactive. This is a generalised error metric that is intended to illustrate that the predictive model is capable of making credible estimates on held out data sets. The limitation of this metric is that it compares a single predicted datapoint to a single real datapoint; a better representation would be to compare shapes of predicted versus real distributions to assess durations above a peak threshold.

TABLE I. PREDICTIVE MODEL SCORES VERSUS LOW CARBON LONDON CUSTOMERS \#1 TO \# 9

\begin{tabular}{|c|c|c|c|c|}
\hline$\#$ & $\begin{array}{c}\text { Maximum } \\
\text { Real } \\
\text { Demand } \\
\text { (kWh) }\end{array}$ & $\begin{array}{c}\text { Mean } \\
\text { Real } \\
\text { Demand } \\
\text { (kWh) }\end{array}$ & $\begin{array}{c}\text { Mean } \\
\text { Absolute } \\
\text { Error } \\
\text { (kWh) }\end{array}$ & $\begin{array}{c}\text { Mean } \\
\text { Absolute Error/ } \\
\text { Mean Real } \\
\text { Demand (\%) }\end{array}$ \\
\hline 1 & 86.1 & 30 & 9.9 & 33 \\
\hline 2 & 32.0 & 9.7 & 3.0 & 31 \\
\hline 3 & 42.4 & 12.9 & 3.9 & 30 \\
\hline 4 & 53.2 & 14.9 & 6.4 & 43 \\
\hline 5 & 111.4 & 37.1 & 9.9 & 27 \\
\hline 6 & 36.9 & 18.6 & 3.4 & 18 \\
\hline 7 & 48.0 & 12.3 & 4.6 & 37 \\
\hline 8 & 38.6 & 26.5 & 5.2 & 20 \\
\hline 9 & 57.7 & 31.0 & 14.0 & 45 \\
\hline
\end{tabular}

\section{Heat Pump Penetration Case Study}

The predictive model is used to analyse a simple power flow scenario for a single $\mathrm{LV}$ feeder with various levels of heat pump penetration. The method described in Section II.B is used to generate simulated heat pump demand profiles for 55 customers and the overall population is scaled according to the method described in Section III.C. This modelled heat pump demand is combined with real smart meter data for the first three months of 2012 [18] which will act as the base load for the $0 \%$ heat pump penetration case.

At present the predictive model only is capable of predicting daily demand figures, whilst the smart meter data is recorded at 30 minute resolution. For this study the daily predicted demand is assumed to be distributed equally across each of the 30-minute daily timeslots. Whilst clearly not representative of a true heat pump profile, this study provides a best-case scenario of heat pump penetration for this population. The heat pump demand averaged across a day is reflective of a perfectly diverse heat pump load; in practice, the demand peaks of a population will be grouped throughout the day and the anticipated peaks will be of larger magnitude than shown in Fig. 6.

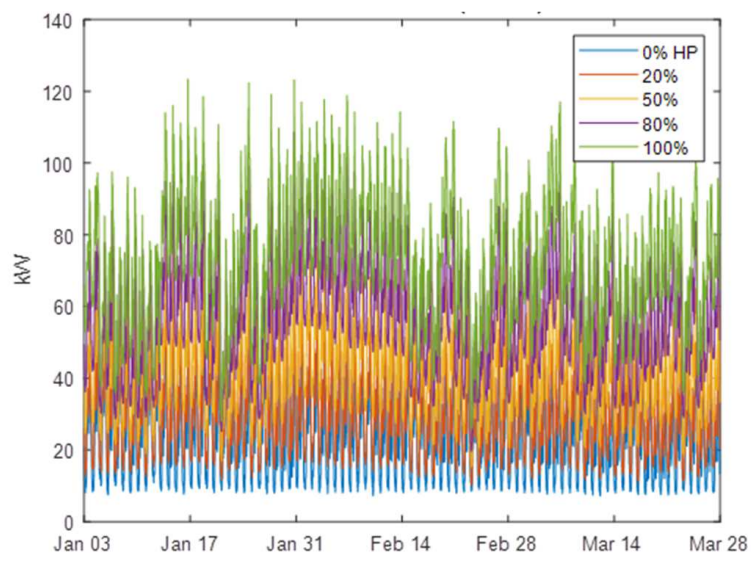

Fig. 6. Total active power for $0-100 \%$ Heat Pump Penetration

The modelled impact of heat pumps at penetrations beyond $20 \%$ is greater than the contribution of the base domestic daily demand at $0 \%$ penetration; for $100 \%$ heat pump penetration on the worst-case cold days the peak power is more than four times the contribution of the base load.

\section{DISCUSSION}

The proposed model in this study offers a simple approach for generating simulated daily heat pump demand for a sample population. The process described is an initial exploratory concept for probabilistically modelling heat pump demand and therefore there are several key areas with opportunity for further development, depending on the final intended application of the model. Broadly these areas can be grouped as refinement of the model scoring metrics, improvement of the normalisation process and adaptation of the model to incorporate sub-daily demand predictions. This section will consider these areas in more detail as well as discuss results and potential model applications.

Whilst relatively large when compared to the mean demand, the MAE error is relatively small for all customers when compared to the maximum heat pump demand. 
Operation of the heat pump at its maximum value is likely to have the greatest risk for any application under study and the proportion of the MAE to the maximum real demand should be considered. Due to the sparsity of data and high variance in demand magnitude at the cold operational extreme, the definition of the Gaussian distributions for cold temperatures could be developed further to accommodate this shortfall with the data.

Despite these limitations, the predictive model provides a good general correlation between the real and predicted demand. Fig. 5 highlights the ability of the predictive model to forecast overall demand trends, although the current limitations of the simple scaling method is visible in offsets at the minimum and maximum operating points. This model can be used to assess the network impact of heat pumps for different geographical locations - the sensitivity of heat pump demand to temperature is critical and this model presents an estimation of the magnitude of this effect.

Realistically the predicted daily demand will be constrained to certain windows of operation rather than evenly spread across 24 hours as is currently modelled. Heat pumps are characterised by low levels of temporal diversity and high heat pump penetration on a network creates the possibility of short periods of high demand which may result in thermal or voltage limits being breached. The primary area for future development would therefore be development of multivariate distributions that account for time as well as temperature. The RHPP dataset features high-resolution 2-minutely demand data and therefore presents an ideal candidate for deriving this.

\section{CONCLUSION}

This paper has defined a probabilistic model for predicting and translating the electrical demand of single heat pump or group of heat pumps across a population. A generic relationship between heat pump electrical demand and temperature has been identified from real customer data and characterised to generate heat pump demand predictions for independent datasets. This model facilitates the analysis of heat pump demand that is sensitive to local temperature conditions, rather than blanket rescaling of existing customer data as has been performed in past literature. This creates future opportunities for examining heat pump demand sensitivity for different geographical locations.

Further development to incorporate sub-daily demand shape profiles and refinement of model error metrics will provide a fully scalable probabilistic heat pump demand model that captures critical demand characteristics of heat pump demand derived from real data. This can then be combined with network models and existing domestic demand data in order to predict the probability that certain network conditions will occur.

\section{REFERENCES}

[1] Ofgem , "Ofgem's Future Insights Series - The Decarbonisation of Heat," 2016. [Online]. Available:

https://www.ofgem.gov.uk/system/files/docs/2016/11/ofgem_future insights_programme_-_the_decarbonisation_of_heat.pdf. [Accessed 05 February 2020]

[2] Catapult Energy Systems, "Living Carbon Free - Exploring with a net-zero target means for households," 2019. [Online]. Available: https://www.theccc.org.uk/wp-content/uploads/2019/06/ESC-LivingCarbon-Free-CCC.pdf. [Accessed 2 February 2020].
[3] M. Ahmadi, M. Ahmadi, M. Sadaghiani, M. Ghazvini, S. Shahriar and M. Alhuyi Nazari, "Ground source heat pump carbon emissions and ground - source heat pump systems for heating and cooling of buildings: A review," Environmental Progress \& Sustainable Energy, vol. 37, no. 4, pp. 1241-1265, 2017.

[4] Committee on Climate Change, "Next steps for UK heat policy," October 2016. [Online]. Available: https://www.theccc.org.uk/wpcontent/uploads/2016/10/Next-steps-for-UK-heat-policy-Committeeon-Climate-Change-October-2016.pdf. [Accessed 2 February 2020].

[5] B. Asare-Bediakoa, W. Klinga and P. Ribeiroab, "Future residential load profiles: Scenario-based analysis of high penetration of heavy loads and distributed generation," Energy and Buildings, vol. 75, pp. 228 - 238, 2014.

[6] C. Hanmer, M. Shipworth, D. Shipworth and E. Carter, "How household thermal routines shape UK home heating demand patterns," Energy Efficiency, no. 12, pp. 5-17, 2019.

[7] Department for Business, Energy \& Industrial Strategy, "Renewable Heat Incentive: A Reformed Sheme," December 2016. [Online] Available:

https://assets.publishing.service.gov.uk/government/uploads/system/ uploads/attachment_data/file/577024/RHI_Reform_Government_res ponse_FINAL.pdf. [Accessed 22 February 2020].

[8] UCL Energy Institute, "Final Report on Analysis of Heat Pump Data from the Rewnewable Heat Premium Payment Scheme," March 2017. [Online]. Available:

https://assets.publishing.service.gov.uk/government/uploads/system/ uploads/attachment_data/file/606818/DECC_RHPP_161214_Final_ Report_v1-13.pdf. [Accessed 22 February 2020].

[9] National Statistics, "RHI monthly deployment data: December 2019 (Annual edition)," 23 January 2020. [Online]. Available: https://assets.publishing.service.gov.uk/government/uploads/system/ uploads/attachment_data/file/859989/RHI_monthly_official_stats_ta bles_Dec_19_final.xlsx. [Accessed 2 February 2020].

[10] D. Jenkins, R. Tucker and R. Rawlings, "Modelling and simulation of a heat pump for simultaneous heating," Energy and Buildings, vol. 41, no. 6, pp. 587-595, 2009.

[11] J. Love, A. Z. Smith, S. Watson, E. Oikonomoua, A. Summerfield, C. Gleeson, P. Biddulpha, L. F. Chiu, J. Wingfield, C. Martin, A. Stone and R. Lowe, "The addition of heat pump electricity load profiles to GB electricity," Applied Energy, vol. 204, pp. 332-342, 2017.

[12] A. Navarro-Espinosa and P. Mancarella, "Probabilistic modeling and assessment of the impact of electric heat pumps on low voltage distribution networks," Applied Energy, vol. 127, pp. 249 - 266, 2014.

[13] UKPN, "Low Carbon London Heat Pump Load Profiles," UK Power Networks, 2017.

[14] Department of Energy and Climate Change, "Renewable Heat Premium Payment Scheme: Heat Pump Monitoring: Cleaned Data, 2013-2015," 2017. [Online]. [Accessed 5 February 2020].

[15] R. Lowe, "Renewable Heat Premium Payment Scheme: Heat Pump Monitoring: Cleaned Data, 2013-2015," Department of Energy and Climate Change, 2017.

[16] Centre for Environmental Data Analysis, "MIDAS Open: UK hourly weather observation data, v201908," Centre for Environmental Data Analysis, 2019.

[17] Durham Energy Intstitute and Element Energy, "CLNR - Insight Report: Domestic Heat Pumps," 23 January 2015. [Online] Available: http://www.networkrevolution.co.uk/wpcontent/uploads/2015/01/CLNR-L091-Insight-Report-DomesticHeat-Pumps.pdf. [Accessed 02 February 2020].

[18] Commission for Energy Regulation (CER), "CER Smart Metering Project - Electricity Customer Behaviour Trial," 2010. [Online]. Available: www.ucd.ie/issda/CER-electricity. [Accessed 24 February 2020]. 